\title{
Editorial:
}

\section{8 special issue on artificial intelligence 2.0: theories and applications}

\author{
Yun-he PAN ${ }^{1,2}$ \\ ${ }^{I}$ Zhejiang University, Hangzhou 310027, China \\ ${ }^{2}$ Chinese Academy of Engineering, Beijing 100088, China \\ E-mail: panyh@cae.cn
}

https://doi.org/10.1631/FITEE.1810000

In July 2017, the Chinese government issued a guideline on developing artificial intelligence (AI), namely, the 'New-Generation Artificial Intelligence Development Plan', through 2030 to the public, setting a goal of becoming a global innovation center in this field by 2030 .

According to the development plan, breakthroughs should be made in basic theories of AI in terms of big data intelligence, cross-media computing, human-machine hybrid intelligence, collective intelligence, autonomous unmanned decisionmaking, brain-like computing, and quantum intelligent computing.

The next-generation AI would be never-ending (self) learning from data and experience, intuitive reasoning and adaptation (Pan, 2016, 2017). From the perspective of overcoming the limitation of existing $\mathrm{AI}$, it is generally recognized that the crossdisciplinary collaboration is a key for AI having real impact on the world.

Thanks for the efforts from researchers in computer science, statistics, robotics, and psychiatry, the topics in this special issue consist mainly of five subjects: (1) fundamental issues in AI such as interpretable deep learning and unsupervised learning (i.e., domain adaptation and generative adversarial learning); (2) brain-like learning such as spiking neural network and memory-augmented reasoning; (3) human-in-the-loop learning such as crowdsourcing design and digital brain with crowd power; (4) creative applications such as social chatbots (i.e., XiaoICe) and automatic speech recognition; (5) Dr. Raj Reddy from $\mathrm{CMU}$ shared his view on the new-generation AI,

(C) Zhejiang University and Springer-Verlag GmbH Germany, part of Springer Nature 2018
Prof. Bin Yu from UC Berkeley advocated that AI should use statistical concepts through humanmachine collaboration, and researchers from the Chinese Academy of Sciences surveyed the acceleration of deep neural networks.

All of interview, perspective, survey, and research papers target rethinking the appropriate ways for a general scenario or a specific application.

In an interview, Dr. Raj Reddy shared his views on the new-generation $\mathrm{AI}$ and detailed the idea of cognition amplifiers and guardian angles (FITEE editorial staff, 2018).

$\mathrm{Yu}$ and Kumbier (2018) discussed how humanmachine collaboration can be approached in AI through the statistical concepts of population, question of interest, representativeness of training data, and scrutiny of results (PQRS). The PQRS workflow provides a conceptual framework for integrating statistical ideas with human input into AI products and research.

Shum et al. (2018) discussed the issue of social chatbots. The design of social chatbots must focus on user engagement and take both intellectual quotient (IQ) and emotional quotient (EQ) into account. Using XiaoIce as an illustrative example, authors introduced key technologies in building social chatbots from core chat to visual sense to skills.

Zhang and Zhu (2018) reviewed recent studies in emerging directions of understanding neuralnetwork representations and learning interpretable neural networks. They revisited visualization of convolutional neural network (CNN) representations, methods of diagnosing representations of pre-trained CNNs, approaches for disentangling $\mathrm{CNN}$ representations, learning of

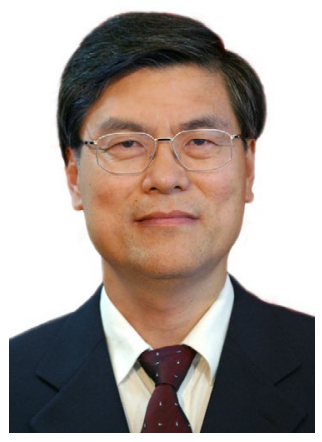

Prof. Yun-he PAN Editor-in-Chief 
interpretable CNNs, and middle-to-end learning based on model interpretability.

Qian et al. (2018) reviewed the cocktail party problem, i.e., tracing and recognizing the speech from a specific speaker when multiple speakers talk simultaneously. This paper focused on the speech separation problem given its central role in the cocktail party environment, and described the conventional single-channel techniques, the conventional multichannel techniques, and the newly developed deep learning based techniques.

Cheng et al. (2018) provided a comprehensive survey about the recent advances on network acceleration, compression, and accelerator design from both algorithm and hardware sides in terms of network pruning, low-rank approximation, network quantization, teacher-student networks, compact network design, and hardware accelerator.

$\mathrm{Xu}$ et al. (2018) proposed a platform of digital brain using crowd wisdom for brain research via the computational AI model of synthetic reasoning and multi-source analogical reasoning.

Li et al. (2018) introduced layer-wise domain correction (LDC), a new unsupervised domain adaptation algorithm which adapts an existing deep network through additive correction layers spaced throughout the network.

Duan et al. (2018) devised an architecture named the temporality-enhanced knowledge memory network (TE-KMN) and applied the model to factoid question answering. The proposed approach not only encoded the temporal cues in a sequence of ordered sentences, but also used the external knowledge to have a better understanding of a given question.

Wang et al. (2018) introduced and investigated the use of generative adversarial networks (GAN) for novelty detection. In training, the GAN learns from ordinary data. Then, using previously unknown data, both the generator and discriminator with the designed decision boundaries can be used to separate novelty from ordinary patterns.

Xiang et al. (2018) introduced a series of studies to show how flexible crowdsourcing design can produce original design ideas consistently. Specifically, they described the typical procedure of flexible crowdsourcing design, the refined crowdsourcing tasks, the factors that affect the idea development process, the method for calculating idea development potential, and two applications of the flexible crowdsourcing design method.

Ma et al. (2018) proposed a spiking neural network (SNN), named MD-SNN, with three key features: (1) using receptive field to encode spike trains from images, (2) randomly selecting partial spikes as inputs for each neuron to approach the absolute refractory period of neuron, and (3) using groups of neurons to make decisions.

We appreciate all the authors who submitted their manuscripts for consideration and those individual reviewers for their timely and professional comments.

\section{References}

Cheng J, Wang PS, Li G, et al., 2018. Recent advances in efficient computation of deep convolutional neural networks. Front Inform Technol Electron Eng, 19(1):64-77. https://doi.org/10.1631/FITEE.1700789

Duan XY, Tang SL, Zhang SY, et al., 2018. Temporalityenhanced knowledge memory network for factoid question answering. Front Inform Technol Electron Eng, 19(1):104-115. https://doi.org/10.1631/FITEE.1700788

FITEE editorial staff, 2018. An interview with Dr. Raj Reddy on artificial intelligence. Front Inform Technol Electron Eng, 19(1):3-5. https://doi.org/10.1631/FITEE.1700860

Li S, Song SJ, Wu C, 2018. Layer-wise domain correction for unsupervised domain adaptation. Front Inform Technol Electron Eng, 19(1):91-103. https://doi.org/10.1631/FITEE.1700774

Ma YQ, Wang ZR, Yu SY, et al., 2018. A novel spiking neural network of receptive field encoding with groups of neurons decision. Front Inform Technol Electron Eng, 19(1): 139-150. https://doi.org/10.1631/FITEE.1700714

Pan YH, 2016. Heading toward artificial intelligence 2.0. Engineering, 2(4):409-413. https://doi.org/10.1016/J.ENG.2016.04.018

Pan YH, 2017. Special issue on artificial intelligence 2.0. Front Inform Technol Electron Eng, 18(1):1-2. https://doi.org/10.1631/FITEE.1710000

Qian YM, Weng C, Chang XK, et al., 2018. Past review, current progress, and challenges ahead on the cocktail party problem. Front Inform Technol Electron Eng, 19(1):4063. https://doi.org/10.1631/FITEE.1700814

Shum HY, He XD, Li D, 2018. From Eliza to Xiaolce: challenges and opportunities with social chatbots. Front Inform Technol Electron Eng, 19(1):10-26 https://doi.org/10.1631/FITEE.1700826

Wang HG, Li X, Zhang T, 2018. Generative adversarial network based novelty detection using minimized reconstruction error. Front Inform Technol Electron Eng, 19(1): 116-125. https://doi.org/10.1631/FITEE.1700786

Xiang W, Sun LY, You WT, et al., 2018. Crowdsourcing intelligent design. Front Inform Technol Electron Eng, 19(1): 126-138. https://doi.org/10.1631/FITEE.1700810

Xu D, Dai F, Lu Y, 2018. A platform of digital brain using crowd power. Front Inform Technol Electron Eng, 19(1): 78-90. https://doi.org/10.1631/FITEE. 1700800

$\mathrm{Yu}$ B, Kumbier K, 2018. Artificial intelligence and statistics. Front Inform Technol Electron Eng, 19(1):6-9. https://doi.org/10.1631/FITEE.1700813

Zhang QS, Zhu SC, 2018. Visual interpretability for deep learning: a survey. Front Inform Technol Electron Eng, 19(1):27-39. https://doi.org/10.1631/FITEE.1700808 